\title{
Niveles de mercurio en los pescados de mayor consumo en los mercados de Tacna exceden límites permitidos por entidades regulatorias
}

\section{Mercury levels in the most consumed fish in Tacna markets exceed limits allowed by regulatory entities}

\author{
Paula Nohelia Ninaja Sarmiento $^{1 \mathrm{a}}$, Ricardo Ortiz Faucheux ${ }^{2 \mathrm{~b}}$ \\ ${ }^{1}$ Investigador independiente. \\ ${ }^{2}$ Escuela de Farmacia y Bioquímica, Facultad de Ciencias de la Salud; Universidad Nacional Jorge Basadre Grohmann.

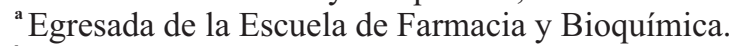 \\ ${ }^{\text {b } Q u i ́ m i c o ~ F a r m a c e ́ u t i c o, ~ D o c t o r ~ e n ~ C i e n c i a s ~ d e ~ l a ~ S a l u d, ~ D o c e n t e ~ U n i v e r s i t a r i o ~ C a ́ t e d r a ~ d e ~ T o x i c o l o g i ́ a ~ y ~ Q u i ́ m i c a ~}$ \\ Legal, Director Escuela de Farmacia y Bioquímica.
}

\begin{abstract}
Resumen
Introducción: El presente trabajo buscó determinar la concentración de mercurio en bonito $(S$. Sarda), caballa $(S$. Scombrus), lisa (M. Cephalus), lorna (S. Deliciosa) y diamante (I. Oxyrinchus); cinco especies de pescado más comercializadas en los principales mercados de la ciudad de Tacna (2019), con el fin de proveer información para las entidades regulatorias de la ciudad. Material y métodos: Se valoraron las concentraciones de mercurio en las muestras de músculo comestible de pescado por medio de espectroscopía de emisión atómica por plasma de acoplamiento inductivo. Resultados: La concentración promedio de mercurio fue de $0.883 \pm 0.104 \mathrm{ppm}$. La especie bonito obtuvo la concentración promedio de mercurio más alta de $1.264 \pm 0.115 \mathrm{ppm}$. No obstante, la especie caballa obtuvo la más baja concentración promedio de $0.639 \pm 0.226$ ppm (IC $95 \%$ 0.057-1.221). Comparado a los Límites Máximos Permisibles (LMP) establecidos por la Administración de Alimentos y Medicamentos (FDA) y el Organismo Nacional de Sanidad Pesquera del Perú, el 43.30 \% excedió dichos límites máximos permisibles de 1.0 ppm. Conclusión: Según los límites máximos permisibles de 0.5 ppm permitidos por la Organización Mundial de la Salud y la Agencia de Protección Ambiental de los Estados Unidos, también de 0.5 ppm; el $73.30 \%$ de las muestras de pescado analizadas excedieron este parámetro de mercurio, generando un verdadero riesgo para la salud.
\end{abstract}

Palabras clave: ICP-OES, mercurio, pescado, Tacna.

\begin{abstract}
Introduction: Bonito (S. Sarda), Caballa (S. Scombrus), Lisa (M. Cephalus), Lorna (S. deliciosa), and Diamante (I. Oxyrinchus), fresh or processed, are five of the most prevalent fish species marketed locally in the city of Tacna, in Peru (2019). In order to provide information for regulatory entities of the city, and biomonitoring programs and assess the potential human health risks, concentrations of mercury $(\mathrm{Hg})$ were determined. Material and methods: Mercury concentrations in the edible fish muscle samples of each species were valued, from three markets located in the city of Tacna via inductively couple plasma atomic emission spectroscopy Results: In all five fish species samples, mercury average concentration was $0.883 \pm 0.104 \mathrm{ppm}$. Bonito got the highest average mercury concentration: $1.264 \pm 0.115$ ppm. However, Caballa species got the lowest average mercury concentration $0.639 \pm 0.226$ ppm (95\% CI $0.057-$ 1.221). According to the established United States Food and Drug Administration (FDA) and the Peruvian National Fisheries Health Agency, Maximum Allowed Limits, 43.30 \% exceeded such maximum allowed limits. Conclusion: According to the World Health Organization and the United States Environmental Protection Agency, $73.30 \%$ of the studied samples of fish exceeded their established Maximum Allowed Limits for mercury. Which is a real health risk. Keywords: ICP-OES, mercury, fish, Tacna.
\end{abstract}




\section{Introducción}

La contaminación alimentaria por metales pesados es un problema persistente en la salud pública actual.

Los metales pesados son parte fundamental de las fuentes antropogénicas provenientes de desechos domésticos, agrícolas e industriales; los cuales, al llegar al mar, son peligrosos para la biota marina.

Los peces tienen la capacidad de almacenar el mercurio $(\mathrm{Hg})$ en su organismo, por lo que son un indicador importante de contaminación, pero también implica que su consumo se puede convertir en un problema de salud para las poblaciones que se alimentan con este recurso.

Los riesgos para la salud derivados del consumo de pescado con niveles elevados de mercurio son un problema prioritario en la salud pública; en vista que la Agencia de Protección Ambiental de los Estados Unidos (EPA) indica que su ingesta está relacionada con daños progresivos en el sistema nervioso central, así como con afecciones pulmonares y renales. Además de generar un invaluable deterioro social ligado al nacimiento de niños con malformaciones, retardo psicomotriz y mental (2-4).

Las consecuencias de la intoxicación masiva por mercurio son de carácter irreversible, y frenan el desarrollo sostenible al imposibilitar la utilización y comercialización de recursos contaminados $(1,2)$.

Tomando en cuenta la predilección por la gastronomía marina en nuestra ciudad, el presente trabajo de investigación buscó determinar los niveles de mercurio en los pescados de mayor consumo de los mercados de Tacna para evidenciar si la población local se encuentra expuesta crónicamente al tóxico, mediante su ingesta.

\section{Material y métodos}

Esta investigación fue de naturaleza observacional, transversal, prospectiva y descriptiva.

Para la determinación de $\mathrm{Hg}$ se pesó, aproximadamente, $0.3 \mathrm{~g}$ de muestra, tomada del músculo dorsal de cada pescado, y fue digerido en HotBlock Digester con $8 \mathrm{ml} \mathrm{de}$ $\mathrm{HNO}_{3}$ a $55{ }^{\circ} \mathrm{C}$ para proceder con reflujo ácido, enjuagándose las paredes del tubo hasta que la solución quedó de color amarillo. Luego de 24 horas, se volvieron a llevar las muestras al digestor, pero ahora con $2 \mathrm{ml} \mathrm{de}$ $\mathrm{HClO}_{4}$ hasta llegar a $55^{\circ} \mathrm{C}$. Se continuó el calentamiento dejando evaporar los ácidos hasta que quedó una solución translúcida. Posteriormente, se diluyeron en $20 \mathrm{ml} \mathrm{de}$ $\mathrm{HNO}_{3}$ y se leyeron en equipo de ICP con método de detección de $\mathrm{Hg}$. Se trabajó a la vez con un blanco, el cual recibió el tratamiento respectivo al tipo de medición.

\section{Resultados}

En la temporada que se realizó el estudio (noviembre y diciembre del 2019), las especies de pescado de mayor comercialización en los mercados Miguel Grau, Dos de Mayo y Santa Rosa de Tacna se muestran en el Gráfico 1. Se trabajó entonces con las cinco especies de mayor consumo: bonito, lisa, lorna, diamante y caballa.

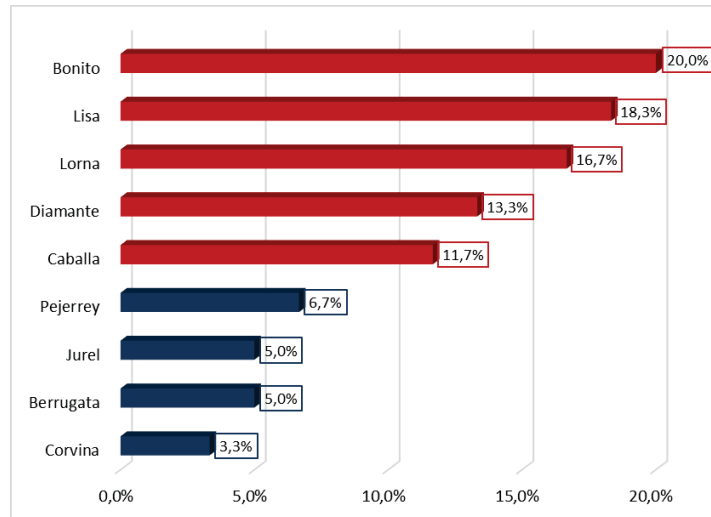

Gráfico 1. Frecuencias de pescados de mayor comercialización en los mercados de Tacna.

Fuente: Encuesta realizada.

Como se muestra en la Tabla 1, la concentración promedio de mercurio entre el total de muestras locales fue de 0.883 \pm 0.104 ppm con un Intervalo de Confianza al $95 \%$ (IC 95 \%) para la media de $0.671-1.096 \mathrm{ppm}$.

Tabla 1. Estadísticos descriptivos de la concentración de mercurio (partes por millón, ppm) de los pescados de mayor comercialización en los mercados de Tacna.

\begin{tabular}{|c|c|c|c|}
\hline \multicolumn{2}{|c|}{ Concentración de mercurio (ppm) } & \multirow{2}{*}{$\begin{array}{c}\text { Estadístico } \\
0,883\end{array}$} & \multirow{2}{*}{$\begin{array}{c}\text { Error Std } \\
0,104\end{array}$} \\
\hline Media & & & \\
\hline \multirow[t]{2}{*}{ IC $95 \%$} & Límite inferior & 0,671 & \\
\hline & Límite superior & 1,096 & \\
\hline \multicolumn{2}{|l|}{ Mediana } & 0,917 & \\
\hline \multicolumn{2}{|l|}{ Varianza } & 0,325 & \\
\hline \multicolumn{2}{|c|}{ Desviación estándar } & 0,570 & \\
\hline \multicolumn{2}{|l|}{ Mínimo } & 0,000 & \\
\hline \multicolumn{2}{|l|}{ Máximo } & 1,830 & \\
\hline
\end{tabular}

Fuente: Matriz de datos procesada en SPSS v.24.

El bonito tiene la concentración promedio de mercurio más alta de $1.264 \pm 0.115 \mathrm{ppm}$. No obstante, la especie caballa es la de más baja concentración promedio de 0.639 \pm 0.226 ppm (IC $95 \% 0.057-1.221)$. Esto se demuestra en la Tabla 2.

Tabla 2. Concentración de mercurio en los pescados; por especie de mayor comercialización en los mercados de Tacna.

\begin{tabular}{lccccc}
\hline \multirow{2}{*}{ Estadisticos } & \multicolumn{5}{c}{ Ppm por especie de pescado } \\
\cline { 2 - 6 } & Lisa & Lorna & Bonito & Diamante & Caballa \\
\hline Media & 0,875 & 0,750 & 1,264 & 0,889 & 0,639 \\
Error estándar & 0,310 & 0,134 & 0,115 & 0,302 & 0,226 \\
$\begin{array}{l}\text { Desviación } \\
\text { estándar }\end{array}$ & 0,760 & 0,329 & 0,281 & 0,739 & 0,554 \\
$\begin{array}{l}\text { Varianza } \\
\quad \text { Limite }\end{array}$ & 0,577 & 0,108 & 0,079 & 0,546 & 0,307 \\
IC 95 $\begin{array}{l}\text { inf } \\
\% \quad \text { Limite }\end{array}$ & 0,078 & 0,405 & 0,969 & 0,113 & 0,057 \\
$\quad$ sup & 1,672 & 1,096 & 1,559 & 1,665 & 1,221 \\
\hline
\end{tabular}

Fuente: Matriz de datos procesada en SPSS v.24. 
Comparado a los límites máximos permitidos establecidos por la FDA y la Agencia Nacional de Sanidad Pesquera del Perú, el $43.30 \%$ excede dichos LMP de 1.0 ppm. Por otro lado, según los LMP de 0.5 ppm permitidos por la OMS y la Agencia de Protección Ambiental de los Estados Unidos, también de 0.5 ppm, el $73.30 \%$ de las muestras de pescado analizadas exceden este parámetro para mercurio, generando riesgo para la salud.

Tabla 3. Frecuencias de pescados de mayor comercialización en los mercados de Tacna según los límites máximos permisibles para mercurio.

\begin{tabular}{lcccc}
\hline \multirow{2}{*}{$\begin{array}{c}\text { Concentración } \\
\text { de mercurio }\end{array}$} & \multicolumn{2}{c}{ FDA - SANIPES } & \multicolumn{2}{c}{ OMS - EPA } \\
\cline { 2 - 5 } & $\mathbf{n}$ & $\boldsymbol{\%}$ & $\mathbf{n}$ & $\%$ \\
\hline No excede & 17 & 56.70 & 8 & 26.70 \\
Excede & 13 & 43.30 & 22 & 73.30 \\
Total & 30 & 100.00 & 30 & 100.00 \\
\hline
\end{tabular}

Fuente: Matriz de datos procesada en SPSS v.24

En el Gráfico 2 se representa los altos porcentajes de muestras que tienen mayor concentración de mercurio que lo recomendado por las autoridades nacionales e internacionales.

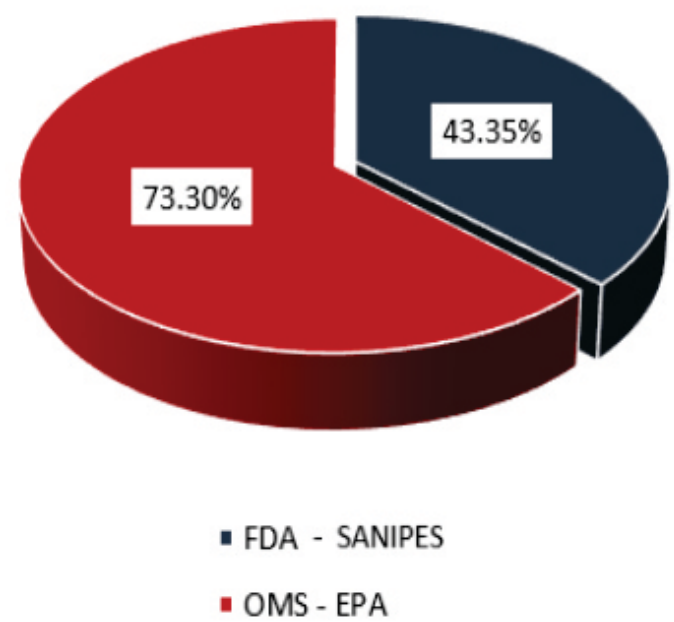

Gráfico 2. Porcentaje de pescados de mayor comercialización en los mercados de Tacna que exceden los LMP para mercurio.

Fuente: Tabla 3.

Se realizó una comparación entre los promedios de concentración de mercurio de las especies de pescado muestreadas, determinándose que todas las especies de pescados superan los LMP de la OMS y EPA $(0.5 \mathrm{ppm})$. La única especie de pescado que supera el LMP de la FDA y SANIPES (1.0 ppm) es el bonito, teniendo como promedio $1.26 \mathrm{ppm}$ de mercurio, como se observa en el Gráfico 3.

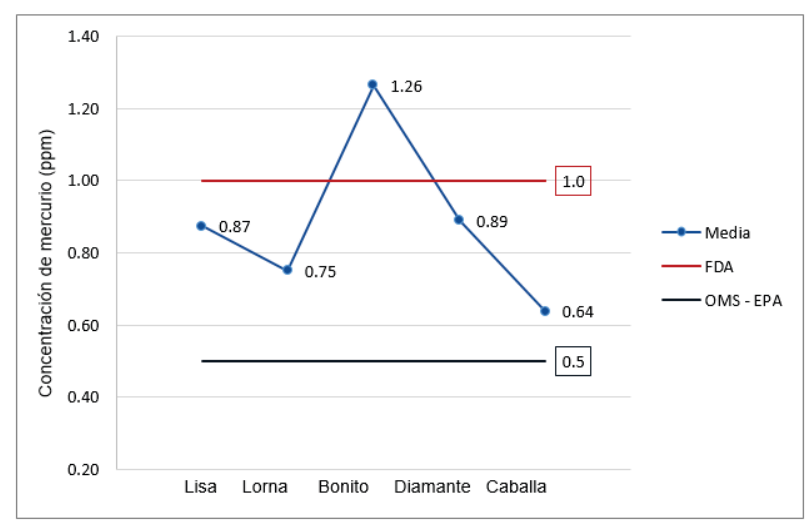

Gráfico 3. Medias de la concentración de mercurio en pescados de mayor comercialización en los mercados de Tacna y los LMP para mercurio.

Fuente: Tabla 2.

En el Gráfico 4 se ilustra que la especie de pescado bonito tiene mayor promedio de mercurio en sus tejidos, presentando $1.265 \mathrm{ppm}$ de mercurio con respecto a las otras especies. Estadísticamente los grupos (especies) no son diferentes; sin embargo, el bonito tiene menor variabilidad (concentraciones de mercurio más cercanos a la media).

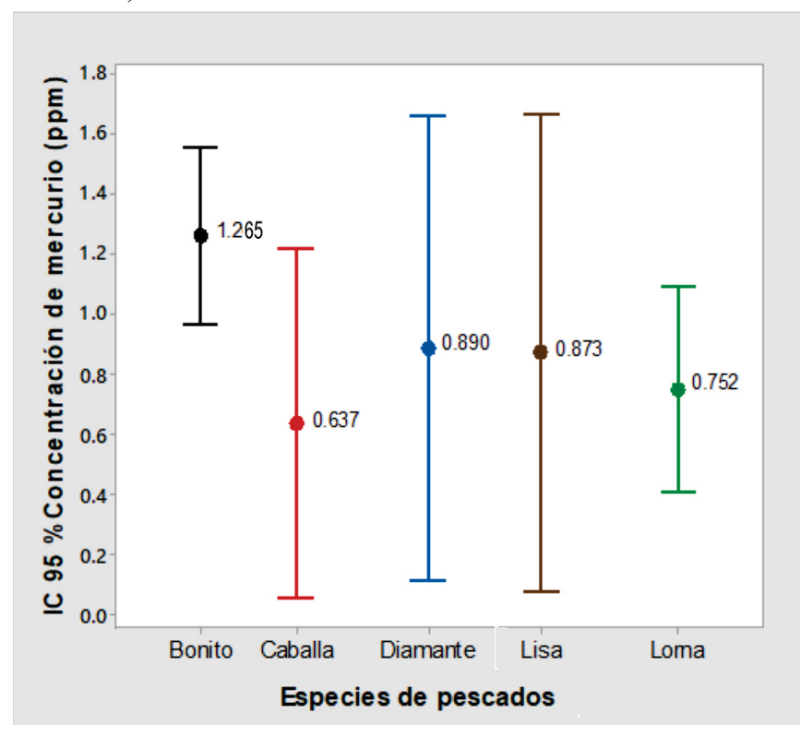

Gráfico 4. IC $95 \%$ para la media de las concentraciones de mercurio en pescados de mayor comercialización según especie marina.

Fuente: Tabla 2.

En el Gráfico 5 se realiza un comparativo entre los mercados Dos de Mayo, Miguel Grau y Santa Rosa de Tacna. Los pescados de mayor comercialización tienen un promedio de mercurio en sus tejidos de $1.018 \mathrm{ppm}$. Por otro lado, teniendo en cuenta el diseño de las barras de error, los tres grupos son estadísticamente iguales en vista 
que las medias de mercurio de los pescados coinciden con la variabilidad de los tres grupos entre sí.

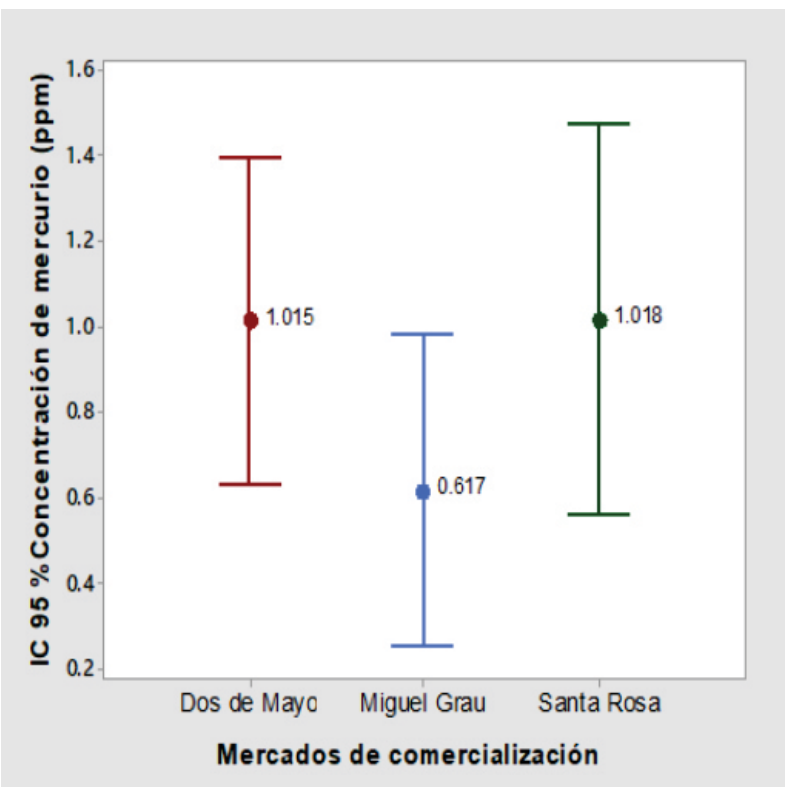

Gráfico 5. IC $95 \%$ para la media de las concentraciones de mercurio en pescados de mayor comercialización según el mercado de venta.

Fuente: Matriz de datos procesada en SPSS v.24.

\section{Discusión}

Mediante una evaluación del Ministerio de la Producción el año 2017, se indicó que en la ciudad de Tacna se ha incrementado el consumo de pescado en un $25 \%$ durante los años 2015 y 2016; siendo junto a Loreto y Ucayali las regiones de mayor consumo anual a nivel nacional(6). En consideración del interés alimentario de la población tacneña por el aumento de consumo de pescados, el presente estudio contribuye con un sustento científico actualizado sobre las especies marinas que prefiere la población tacneña, así como el análisis toxicológico de mercurio en las muestras de mayor frecuencia de comercialización en los mercados.

La procedencia de los pescados de mayor comercialización de los mercados de Tacna son los puertos de Ilo y Morro Sama; ambas localidades tienen cercanía con las minas de Toquepala, Cuajone y la refinería de Ilo, lo cual se ha visto reflejado en las concentraciones de mercurio en los pescados obtenidos del lugar; además se debe considerar que, por otro lado, en Perú, la minería aurífera informal, en un primer momento, estuvo concentrada en Madre de Dios, Puno, Sur Medio y La Libertad como una forma de subsistencia en minas abandonadas o no cerradas; según el informe "Conservación y uso sostenible de ecosistemas para la provisión de servicios ecosistémicos" del Ministerio del Ambiente publicado en enero de 2017. Sin embargo, a partir de mediados de la década pasada, las actividades de minería informal e ilegal se expandieron por todo el territorio peruano. Para el 2013, señala el estudio del Ministerio del Ambiente, esta actividad ya estaba presente en 25 regiones del país $(7,8)$.

En un estudio de Bellido (9) en 2015, menciona que los pescados de mayor consumo en nuestra localidad en el momento del estudio eran el bonito, jurel y pejerrey. Sin embargo, en actuales resultados las especies de mayor comercialización y consumo en los meses de noviembre y diciembre son: lisa $(18.33 \%)$, bonito $(20 \%)$ y lorna $(16.67 \%)$.

En este estudio se obtuvo un valor máximo en las muestras de $1.830 \mathrm{ppm}$ de mercurio. Este valor difiere y supera el valor máximo obtenido de $1.570 \pm 0.22 \mathrm{ppm}$ por el Ministerio de Salud de Chile (MINSAL) en 2012 (10), en un estudio realizado en Valparaíso; por otra parte, el valor obtenido es menor en comparación al estudio de Trujillo et al. (11) en 2005, quienes hallaron una concentración máxima de mercurio inorgánico en pescados de interés comercial de 3,44 ppm. Con referencia a las especies de pescado, el bonito es el que mayor media de concentración de mercurio presentó en las muestras analizadas: $1.264 \pm$ 0.115 ppm (IC 95 \%: 0.969 - 1.559) en comparación con las otras especies. Por otro lado, la especie de pescado caballa es la que menor promedio de concentración de mercurio presentó $0.639 \pm 0.226$ ppm (IC $95 \% 0.057-$ 1.221).

En el estudio de Bedregal, Edwin et al. (12) en 2014, se mostró promedios de concentración de mercurio en la especie de pescado bonito en la ciudad de Arequipa de 8.094, 14.836 y 37.07 ppm, resultados diferentes a los obtenidos en la presente investigación; por otro lado, la investigación de Espinoza, Diego (13) en la ciudad de Tumbes encuentra niveles de mercurio en pescados de interés alimentario inferiores a los hallados en el presente estudio, siendo la concentración máxima de mercurio de $0.112 \mathrm{ppm}$ (especie periche).

En el mercado Santa Rosa de Tacna los pescados de mayor comercialización tienen un promedio de mercurio en sus tejidos de $1.018 \mathrm{ppm}$. Sin embargo, considerando el resultado mostrado por el Gráfico 5 , se puede afirmar matemáticamente que los tres mercados tienen promedios de mercurio (en sus muestras de interés comercial) estadísticamente iguales, porque coinciden en la proximidad de sus promedios.

Teniendo en cuenta los efectos fisiológicos negativos del $\mathrm{Hg}$, se consultó con un especialista en neurología del hospital Hipólito Unanue de Tacna sobre principales problemas de salud en esta área, reportando que los casos neurológicos y cardiovasculares pueden ser no registrados en su totalidad, debido a que no se le considera como causa frecuente de enfermedades como autismo, ni de síntomas neurales: deficiencias visuales y auditivas, retraso mental, pérdida de la memoria y trastornos del lenguaje. Es 
necesario tener un registro de este tipo de intoxicaciones, que pueden pasar inadvertidas por el simple hecho de que en los hospitales no son descartadas; no se hace un análisis ni seguimiento toxicológico por parte del especialista (farmacéutico).

Definitivamente es necesario solicitar a las autoridades competentes que se realice un mayor control a la carne de pescado; pues actualmente, solo se hace un control organoléptico, mas no microbiológico ni toxicológico. Es un derecho de los consumidores tener la seguridad alimentaria.

Es un derecho y también un deber de nosotros como ciudadanos estar informados sobre los riesgos de ingerir alimentos contaminados con metales pesados.

Se concluye que las especies de pescados de mayor comercialización en los mercados de Tacna (Miguel Grau, Dos de Mayo y Santa Rosa) son: lisa (18.33 \%), bonito $(20.00 \%)$, diamante $(13.33 \%)$, caballa (11.67\%) y lorna $(16.67 \%)$. Las demás especies no son altamente comercializadas. El nivel de mercurio en los pescados de mayor comercialización en los mercados de Tacna es de $0.883 \pm 0.104 \mathrm{ppm}$ (IC $95 \% 0.671-1.096 \mathrm{ppm}$ ). El valor máximo encontrado en las muestras de pescados fue de $1.830 \mathrm{ppm}$. La media de la concentración de mercurio de los pescados de mayor comercialización en los mercados de Tacna es distinta y excede al Límite Máximo Permitido (LMP) establecido por la OMS y la EPA. Se determinó con un $\mathrm{p}$ valor de 0.447 que las concentraciones de mercurio de los pescados de mayor comercialización no son estadísticamente diferentes según la especie marina.
Se determinó con un p valor de 0.198 que las concentraciones de mercurio no son estadísticamente diferentes según el mercado de comercialización o distribución. Las especies marinas de mayor consumo de la ciudad de Tacna están contaminadas con Mercurio.

Se recomienda realizar periódicamente más estudios de determinación toxicológica de mercurio orgánico en pescados que abastecen a los mercados de Tacna. Comunicar a los profesionales de la salud y población en general sobre los pescados que tengan mayor cantidad de mercurio que se comercializan en los mercados de Tacna con el fin de prevenir la ingesta de concentraciones elevadas de mercurio inorgánico, y así evitar enfermedades crónicas y congénitas tales como ocurre en las mujeres gestantes. La recomendación aconseja de 2 a 3 porciones de los pescados de baja concentración de mercurio por semana, o un máximo de 340 gramos; sin embargo, todos los peces contienen al menos rastros de mercurio, que pueden ser perjudiciales para el cerebro y el sistema nervioso, si una persona se expone a demasiada cantidad en el tiempo. El máximo nivel de consumo aconsejado en la recomendación final (5) coincide con el nivel anterior recomendado de 12 onzas o 340 gramos por semana. La nueva recomendación coincide con las pautas alimentarias para estadounidenses de 2015-2020. Para los adultos, una porción típica es de 4 onzas, o aproximadamente 100 gramos de pescado, pesado crudo. Los tamaños de la porción para los niños deben ser más pequeños y ajustarse según la edad y la necesidad calórica total. Se recomienda que los niños coman pescado, una o dos veces por semana, seleccionado de entre una variedad de clases de pescados.

\section{Referencias bibliográficas}

1. Fundación Vasca para la Seguridad Agroalimentaria. Elika.net. [Online].; 2005 [cited 2018 setiembre 2. Available from: http://www.elika.net/datos/riesgos/Archivo_EN 6/Mercurio\%20en\%20pescado\%202005.pdf.

2. Bello Moreira I, Gonzalo Vera C, Vera Delgado H. Determinación de mercurio en enlatados de Atún comercial de la Ciudad de Manta, Provincia de Manabi - Ecuador. Alimentos Hoy. 2016 abril; 23(36).

3. García González G. cuantificación de metales pesados en alimentos. Tesis de Grado. Cuautitlán: Universidad Nacional Autónoma de México, Facultad de Estudios Superiores; 2010.

4. USEPA. A decade of tribal environmental health research: results and impacts from EPA's extramural grants and fellowship programs. U.S.A; 2004.
5. Administración de Medicamentos y Alimentos. FDA. Exposición a mercurio en pescados y mariscos durante la etapa gestacional. SafeFood. 2004;(16).

https://www.fda.gov/news-events/comunicados-deprensa/la-fda-y-la-epa-emiten-recomendacionfinal-sobre-el-consumo-de-pescado

6. Ministerio de la Producción. PRODUCE. Consumo per cápita de pescado a nivel nacional. Lima: Ministerio de la Producción, Departamento de Estadística y Mercado; 2017.

7. Ambiente Md. [Online].; 2018 [cited 2018. Available from: http:/www.minam.gob.pe/wpcontent/uploads/2017/05/Anexo-02-PP-1442018.compressed.pdf.

8. Sierra Y. Mongabay. [Online].; 2018 [cited 2018. A v a i l a b 1 e f r o m : https://es.mongabay.com/2018/02/perucontaminacion-por-mercurio/.

9. Bellido Boza L. Consumo de pescados en la 
población Tacneña. Diario Correo. 2015.

10. Ministerio de Salud de Chile. MINSAL. Mercurio en pescados. Revista Médica de Chile. 2014; II(10).

11. Trujillo GF DMLCPL. Evaluación de las concentraciones de mercurio en peces de interés comercial en ecosistemas acuáticos de la Orinoquia. Biodiversidad de la Cuenca. 2010; III (12).
12. Bedregal Vera Edwin Steven NSP NCS RACEG JAVS. Identificación y cuantificación de Mercurio, Cadmio y Plomo en los pescados de mayor consumo de Arequipa. Universidad Católica de Santa María. 2014.

13. Espinoza Ortiz D. Niveles de mercurio, cadmio, plomo y arsénico en peces del río Tumbes y riesgos para salud humana por su consumo. Instituto de Investigación RIIGEO. 2015 diciembre.

\section{Correspondencia:}

rortizf@unjbg.edu.pe
Fecha de recepción: 05 de mayo del 2020

Fecha de aceptación: 09 de junio del 2020 